\title{
RESISTÊNCIA BIOLÓGICA E COLORIMETRIA DA MADEIRA TERMORRETIFICADA DE DUAS ESPÉCIES DE EUCALIPTO ${ }^{1}$
}

\author{
Marília Lazarotto ${ }^{2 *}$, Sérgio da Silva Cava ${ }^{3}$, Rafael Beltrame $^{4}$, Darci Alberto Gatto ${ }^{4}$, André Luiz Missio ${ }^{5}$, \\ Letícia Grellert Gomes ${ }^{6}$ e Taline Rodrigues Mattoso ${ }^{6}$
}

\footnotetext{
${ }^{1}$ Recebido em 26.01.2015 aceito para publicação em 20.11.2015.

${ }^{2}$ Universidade Federal do Rio Grande do Sul, Faculdade de Agronomia, Departamento de Horticultura e Silvicultura, Porto Alegre, RS - Brasil. E-mail: <marilia.lazarotto@ufrgs.br>.

${ }^{3}$ Universidade Federal de Pelotas, Centro de Desenvolvimento Tecnológico, Curso de Engenharia de Materiais, Pelotas, RS - Brasil. E-mail:<sergiocava@gmail.com>.

${ }^{4}$ Universidade Federal de Pelotas, Centro de Engenharias, Curso de Engenharia Industrial Madeireira, Pelotas, RS - Brasil. E-mail: < beltrame.rafael@yahoo.com.br $>$ e $<$ darcigatto@yahoo.com $>$.

${ }^{5}$ Universidade Federal de Santa Maria, Centro de Ciências Rurais, Programa de Pós-graduação em Engenharia Florestal, Santa Maria, RS - Brasil. E-mail: <andreluizmissio@gmail.com>.

${ }^{6}$ Universidade Federal de Pelotas, Graduando em Engenharia Industrial Madeireira, Pelotas, RS - Brasil. E-mail:

$<$ leticiaggomes@hotmail.com>e <nineyrafa@gmail.com>.

*Autor para correspondência.
}

\begin{abstract}
RESUMO - Os objetivos deste estudo foram os seguintes: a) observar o efeito de termorretificação sobre a durabilidade natural da madeira de Eucalyptus tereticornis e Corymbia citriodora após ataque de fungos causadores de podridão-branca (Trametes versicolor e Ganoderma applanatum); b) avaliar parâmetros colorimétricos após a submissão das madeiras a tratamentos térmicos e aos fungos $T$. versicolor e $G$. applanatum. Para realização do estudo, corpos de prova de ambas as espécies foram submetidos aos tratamentos: $\mathrm{T}_{1}$ - testemunha; $\mathrm{T}_{2}-$ autoclave $\left(120^{\circ} \mathrm{C}\right)$ a $1,5 \mathrm{kgf} / \mathrm{cm}^{2}$ durante $1 \mathrm{~h} ; \mathrm{T}_{3}$ - estufa laboratorial $\left(180^{\circ} \mathrm{C}\right)$ por $4 \mathrm{~h}$; e $\mathrm{T}_{4}$ - térmico combinado $\left[120^{\circ} \mathrm{C}(1 \mathrm{~h})+180^{\circ} \mathrm{C}(4 \mathrm{~h})\right]$. Posteriormente, os corpos de prova foram submetidos ao apodrecimento acelerado, sendo expostos às duas espécies de fungos por 16 semanas. Após esse período, foram avaliadas a perda de massa e as variáveis colorimétricas, através dos parâmetros $\mathrm{L}^{*}, \mathrm{a}^{*}, \mathrm{~b}^{*}, \mathrm{C}^{*} \mathrm{e} \mathrm{h}^{\circ}$ antes e depois do ataque pelos fungos. De acordo com os resultados, E. tereticornis e $C$. citriodora foram classificados como altamente resistentes a fungos apodrecedores, exceto no tratamento testemunha de C. citriodora submetido ao ataque de G. applanatum, o qual foi classificado como resistente. Com os tratamentos $\mathrm{T}_{3}$ e $\mathrm{T}_{4}$, a perda de massa foi reduzida em ambas as espécies de madeira. As maiores mudanças nos parâmetros colorimétricos ocorreram devido ao tratamento térmico e à pouca variação observada com o ataque dos fungos apodrecedores. Houve redução da variável claridade ( $\left.L^{*}\right)$ e queda das matrizes vermelho $\left(a^{*}\right)$ e amarelo $\left(b^{*}\right)$, razão por que o tratamento térmico mostrou-se como alternativa para proteção e escurecimento da madeira de eucalipto, tornando-a mais próxima de padrões de coloração de madeiras nobres.
\end{abstract}

Palavras-chave: Podridão branca; Tratamento térmico; Claridade.

\section{BIOLOGICAL RESISTANCE AND COLORIMETRY OF HEAT TREATED WOOD OF TWO EUCALYPTUS SPECIES}

\begin{abstract}
The objectives of this study were: a) to observe the effect of heat treatment on natural durability of Eucalyptus tereticornis and Corymbia citriodora wood to attack of white rot fungi (Trametes versicolor and Ganoderma applanatum); b) to evaluate colorimetric parameters after the submission of the woods to heat treatments and T. versicolor and G. applanatum fungi. To start the study, samples of both specie were submitted to the treatments: $T_{1}$-control; $T_{2}$-autoclave $\left(120^{\circ} \mathrm{C}\right)$ at $1,5 \mathrm{kgf} / \mathrm{cm}^{2}$ for $1 \mathrm{~h}$; $T_{3}$ - oven $\left(180^{\circ} \mathrm{C}\right)$ for 4 hours; and $T_{4}$-thermal combined $\left[120^{\circ} \mathrm{C}(1 \mathrm{~h})+180^{\circ} \mathrm{C}(4 \mathrm{~h})\right]$.
\end{abstract}


Subsequently, the samples were subjected to accelerated decay, being exposed to both species of fungi for 16 weeks. After this period, the mass loss and the colorimetric variables through the parameters $L^{*}, a^{*}, b^{*}$, $C^{*}$ and $h^{\circ}$ were evaluated before and after the fungal attack. According to the results, E. tereticornis and C. citriodora were classified as highly resistant to decay fungi, except for the control treatment of $\boldsymbol{C}$. citriodora subject to the attack of $\boldsymbol{G}$ applanatum, which was classified as resistant. $T_{3}$ and $T_{4}$ treatments reduced the mass loss for both wood species. The biggest changes in colorimetric parameters occur due to heat treatment and minimum variation was observed with the attack of decay fungi. There was a reduction in the variable lightness $\left(L^{*}\right)$ and red $\left(a^{*}\right)$ and yellow $\left(b^{*}\right)$ matrix, thereby the heat treatment could be an alternative to protection and darkening of eucalyptus wood approximating these to hardwood patterns.

Keywords: White rot; Heat treatments; Lightness.

\section{INTRODUÇÃO}

A escassez das madeiras nativas de alta durabilidade natural nas florestas de produção resulta na elevação do valor comercial para o mercado consumidor (ARAÚJO et al., 2012). Por isso, a utilização de espécies introduzidas, provenientes de reflorestamentos, torna-se alternativa viável e sustentável, desde que se consiga alta qualidade em relação a parâmetros físico-mecânicos e biológicos (preservação). Espécies do gênero Eucalyptus apresentam características silviculturais desejáveis, como incremento, forma e desrama natural. Além disso, a grande diversidade de espécies possibilita a sua adaptação às diversas condições edafoclimáticas, sendo a versatilidade de propagação, que é facilmente obtida por sementes ou por via vegetativa, outro facilitador (BÔAS et al., 2009).

Conforme Poubel et al. (2013), um dos maiores desafios para os pesquisadores na área de tecnologia da madeira têm sido encontrar soluções para a instabilidade dimensional e durabilidade natural da madeira. O desenvolvimento de pesquisas sobre preservantes da madeira passa por uma fase crucial, sendo necessários o seu direcionamento para análise de produtos com menor potencial de danos ambientais e a saúde de todos os organismos (BRAND et al., 2006). Conforme Weiland e Guyonnet (2003), a utilização de tratamento térmico provoca alteração da composição química da madeira, o que pode melhorar a resistência ao ataque de fungos. Nesse tipo de tratamento ocorrem degradação das hemiceluloses (ESTEVES; PEREIRA, 2009), aumento da proporção de celulose cristalina e geração de novos compostos extraíveis (ESTEVES et al., 2008), além do incremento das ligações cruzadas da rede de lignina (NUOPPONEN et al., 2004; TJEERDSMA; MILITZ, 2005). Essas mudanças reduzem o teor de umidade de equilíbrio (CADEMARTORI et al., 2014b); geram novos extrativos que podem atuar como fungicidas; modificam quimicamente os polissacarídeos e lignina da madeira, o que leva à inibição das enzimas digestivas dos fungos; degradam as hemiceluloses, que constituem uma das principais fontes de nutrientes para os fungos; e, por fim, aumentam a hidrofobia da madeira (SILVA et al., 2008).

Na Europa, Estados Unidos e Canadá, a madeira tratada termicamente é comercializada em processos patenteados: Retification ${ }^{\circledR}$ (França), Bois Perdure ${ }^{\circledR}$ (França e Canadá), Oil Heat Treatment-OHT ${ }^{\circledR}$ (Alemanha), Plato ${ }^{\circledR}$ Wood (Países Baixos),

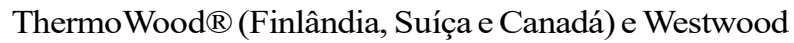
(Estados Unidos, Canadá e Rússia) (MILITZ, 2002). Além da maior durabilidade natural e estabilidade dimensional, a madeira termorretificada apresenta outras características, como: em geral, escurecimento da madeira, perda de massa, alterações químicas, maior capacidade de isolação térmica e não requer a utilização de preservativos químicos, o que representa uma vantagem do ponto de vista ambiental (KAMDEM et al., 2002). Delucis et al. (2014) verificaram aumento da estabilidade dimensional da madeira de Eucalyptus botryoides, Corymbia citriodora, E. paniculata e E. tereticornis após a termorretificação a $200{ }^{\circ} \mathrm{C}$.

Além dos efeitos benéficos, em termos de durabilidade e estabilidade dimensional, Zanuncio et al. (2014) ainda salientaram que o processo de termorretificação pode alterar a cor da madeira de Eucalyptus, fazendo que esta se aproxime das madeiras de maior valor de mercado, processo muito utilizado nos Estados Unidos e na Europa e que, no Brasil, ainda é inexpressivo.

Diante do exposto, os objetivos deste trabalho foram: a) verificar o efeito de diferentes tratamentos térmicos sobre a durabilidade natural da madeira de Eucalyptus tereticornis e Corymbia citriodora ao ataque 
de fungos causadores de podridão-branca (Trametes versicolor e Ganoderma applanatum); b) avaliar mudanças em parâmetros colorimétricos após a submissão das madeiras de E. tereticornis e C. citriodora aos tratamentos térmicos e aos fungos causadores de podridão-branca.

\section{MATERIAL E MÉTODOS}

\subsection{Obtenção e preparo do material}

Foram utilizados corpos de prova com dimensões de 9,0 x 25,0 x 25,0 mm (menor dimensão no sentido axial da madeira) de duas espécies: Eucalyptus tereticornis e Corymbia citriodora provenientes de árvores com aproximadamente 60 anos de idade. Os corpos de prova tiveram a massa específica aparente a $12 \%$, determinada a partir da sua massa e volume obtidos após a estabilização da umidade de equilíbrio delas em câmara climatizada. Para cada tratamento, quatro corpos de prova de cada espécie foram utilizados.

Foi avaliada a resistência da madeira ao apodrecimento acelerado ao ataque de duas espécies fúngicas, causadoras de podridão-branca da madeira: Trametes versicolor e Ganoderma applanatum. Os isolados fúngicos foram cedidos pelo Setor de Biodegradação e Preservação da Madeira - Laboratório de Produtos Florestais (LPF) do Instituto Brasileiro do Meio Ambiente e dos Recursos Naturais Renováveis (IBAMA), localizado em Brasília, DF. Os isolados foram armazenados em câmara incubadora tipo Biological Oxygen Demand (BOD) sob temperatura de $25 \pm 3{ }^{\circ} \mathrm{C}$ sem controle de umidade relativa e na ausência de luz.

\subsection{Montagem do ensaio de apodrecimento acelerado}

O ensaio de apodrecimento acelerado foi conduzido conforme os procedimentos da ASTM D 2017 (AMERICAN SOCIETY FOR TESTING AND MATERIALS - ASTM, 2005).

Para a montagem do experimento foram utilizados frascos de vidro com capacidade para $500 \mathrm{~mL}$ preenchidos com 100 g de solo, com pH 6,0 e capacidade de retenção de $40 \%$. Após a determinação do teor de umidade do solo, a capacidade de retenção do solo foi ajustada para $130 \%$, com adição de água destilada. Para cada tratamento, foram utilizados quatro frascos (repetições), os quais receberam, cada um, uma placa-suporte confeccionada com alburno da espécie Pinus elliottii nas dimensões de 3,0 x 29,0 x 35,0 mm, sendo a maior dimensão paralela à grã. Essa placa serviu como substrato para o estabelecimento inicial da colônia fúngica. Depois de montados, os frascos foram submetidos a duas autoclavagens de $40 \mathrm{~min}$, com intervalo de $24 \mathrm{~h}$, à temperatura de $120^{\circ} \mathrm{C}$ e pressão de $1 \mathrm{~atm}$.

Em câmara de fluxo laminar (ambiente asséptico), fez-se a repicagem das colônias fúngicas, com um disco de $5 \mathrm{~mm}$ da colônia sobre as placas-suporte de $P$. elliottii. Esses frascos ficaram incubados por 30 dias em câmara climatizada $\left(20{ }^{\circ} \mathrm{C}\right.$ e $65 \%$ de umidade relativa), sem luminosidade, para o desenvolvimento dos fungos sob a placa suporte.

\subsection{Tratamentos térmicos}

Os tratamentos térmicos foram realizados a partir da utilização de uma autoclave vertical de esterilização (condições úmidas) e de uma estufa laboratorial (condições secas em atmosfera com presença de ar). Os tratamentos foram compostos da seguinte forma:

- $\mathrm{T}_{1}$ (testemunha) - sem tratamento, apenas esterilização em autoclave.

$-\mathrm{T}_{2}\left(120^{\circ} \mathrm{C}-1 \mathrm{~h}\right)-$ tratamento em autoclave $(120$ ${ }^{\circ} \mathrm{C}$ ), realizado com pressão nominal de $1,5 \mathrm{kgf} / \mathrm{cm}^{2}$ durante $1 \mathrm{~h}$.

$-\mathrm{T}_{3}\left(180^{\circ} \mathrm{C}-4 \mathrm{~h}\right)$ - tratamento térmico em estufa laboratorial $\left(180^{\circ} \mathrm{C}\right)$, realizado em equipamento elétrico provido de controle termostático de temperatura. As condições estabelecidas para este processo foram sem circulação forçada de ar, durante $4 \mathrm{~h}$, e corpos de prova em equilíbrio higroscópico como condição inicial de tratamento.

$-\mathrm{T}_{4}$ (combinado) - tratamento térmico combinado $\left[120{ }^{\circ} \mathrm{C}(1 \mathrm{~h})+180{ }^{\circ} \mathrm{C}(4 \mathrm{~h})\right]$. Após o tratamento em autoclave, os corpos de prova foram dispostos em câmara climática até a realização do tratamento térmico em estufa laboratorial.

Os corpos de prova tratados termicamente $\left(\mathrm{T}_{2}\right.$, $\mathrm{T}_{3}$ e $\mathrm{T}_{4}$ ) foram condicionados em câmara climática por aproximadamente 30 dias e, antes de serem postos em contato com os fungos, foram secos em estufa na temperatura de $50^{\circ} \mathrm{C}$ até massa constante, para obtenção da massa inicial. Depois, eles foram identificados, envolvidos em papel-filtro e autoclavados por $40 \mathrm{~min}$ a $120^{\circ} \mathrm{C}$, para esterilização, para o que os corpos de prova foram colocados em frascos de vidro fechados, a fim de evitar a entrada de vapor.

Revista Árvore, Viçosa-MG, v.40, n.1, p.135-145, 2016

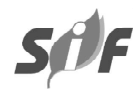


Após a esterilização, os corpos de prova foram introduzidos nos frascos e colocados em contato com a placa-suporte completamente colonizada pelo fungo, permanecendo nessa condição por um período de 16 semanas.

\subsection{Avaliação do ensaio de apodrecimento acelerado}

Após o período de 16 semanas, os corpos de prova foram retirados dos frascos para remoção dos micélios com o auxílio de pincel em câmara de fluxo laminar e novamente colocados para secar em estufa na temperatura de $50^{\circ} \mathrm{C}$ até massa constante, para obtenção de suas massas finais.

A resistência natural da madeira das espécies foi determinada em função da perda de massa, como mostrado na Tabela 1 .

\subsection{Colorimetria}

O ensaio foi realizado com o auxílio de um colorímetro portátil Konica Minolta, modelo CR-400, com abertura do sensor de $8 \mathrm{~mm}$. O aparelho foi configurado para o uso de fonte de luz (iluminante) D65 e ângulo de observação de segundos, no padrão descrito pela Commission Internationale de L'éclairage (CIE-L*a*b*). Para cada um dos corpos de prova, realizaram-se duas verificações em cada no planto transversal (plano de dimensão de $25 \mathrm{~mm}$ ). Obtiveram-se os parâmetros colorimétricos $\mathrm{L}^{*}, \mathrm{a}^{*} \mathrm{e} \mathrm{b*}$, os quais indicam claridade (preto, 0 - branco, 100), coordenada cromática vermelho $(+)$ - verde (-) e coordenada cromática amarelo (+) azul (-), respectivamente, e, ainda, $\mathrm{C}^{*} \mathrm{e} \mathrm{h}^{\circ}$, os quais indicam saturação de cor e ângulo de tinta, respectivamente.

A variação colorimétrica foi realizada nos corpos de prova antes e depois da submissão destes ao ensaio de apodrecimento acelerado, a fim de verificar a variação colorimétrica em função dos tratamentos térmicos e

Tabela 1 - Classes de resistência ao apodrecimento segundo a Norma ASTM D 2017 (2005).

Table 1 -Classes to wood decay according to the standard ASTM D 2017 (2005).

\begin{tabular}{lcc}
\hline Classe de resistência & $\begin{array}{c}\text { Perda de } \\
\text { massa (\%) }\end{array}$ & $\begin{array}{c}\text { Massa } \\
\text { residual (\%) }\end{array}$ \\
\hline Alta Resistência (AR) & $0-10$ & $90-100$ \\
Resistente (R) & $11-24$ & $76-89$ \\
Resistência Moderada (RM) & $25-44$ & $56-75$ \\
Não Resistente (NR) & $>45$ & $>55$ \\
\hline
\end{tabular}

Revista Árvore, Viçosa-MG, v.40, n.1, p.135-145, 2016 da ação dos fungos causadores da podridão-branca sobre esses tratamentos.

\subsection{Análise estatística}

O experimento foi instalado segundo o delineamento inteiramente casualizado, com quatro repetições de cada tratamento. $\mathrm{O}$ experimento foi trifatorial: espécies de eucalipto $x$ espécies fungos $x$ tratamentos térmicos $(2 \times 2 \times 4)$, totalizando 16 tratamentos e, portanto, 64 corpos de prova. As porcentagens de perda de massa e os parâmetros colorimétricos foram submetidos à análise de variância para verificar a existência de diferenças significativas, pelo teste de F, com o auxílio do programa Sisvar, versão 5.3 (FERREIRA, 2011). O teste de médias aplicado foi o de Tukey a $5 \%$ de significância.

\section{RESULTADOS}

A massa específica, determinada a $12 \%$ de umidade para Eucalyptus tereticornis, foi de $0,873 \mathrm{~g} / \mathrm{cm}^{3}$ e para Corymbia citriodora, $1,026 \mathrm{~g} / \mathrm{cm}^{3}$.

A interação trifatorial não foi significativa, por isso as médias de massa residual e perda de massa foram avaliadas separadamente para cada uma das espécies de eucalipto. Para a madeira de E. tereticornis, todos os tratamentos, incluindo a testemunha, tiveram a madeira classificada como altamente resistente a fungos xilófagos, com massa residual superior a $90 \%$ e perda de massa inferior a 10\% (Tabela 2). A interação entre espécies fúngicas testadas e tratamentos foi significativa $(p<0,05)$, e somente na testemunha houve diferença substantiva, sendo a massa residual inferior quando a madeira foi exposta ao fungo G. applanatum. Nos tratamentos $\mathrm{T}_{2}\left(120^{\circ} \mathrm{C}-1 \mathrm{~h}\right), \mathrm{T}_{3}\left(180^{\circ} \mathrm{C}-4 \mathrm{~h}\right)$ e $\mathrm{T}_{4}$ (combinado), a massa residual encontrada em $T$. versicolor foi superior, em ambas as espécies, ao tratamento controle. Já no fungo G. applanatum os melhores resultados foram encontrados nos tratamentos $\mathrm{T}_{3}\left(180^{\circ} \mathrm{C}-4 \mathrm{~h}\right)$ e $\mathrm{T}_{4}$ (combinado), evidenciando a influência positiva da temperatura de $180{ }^{\circ} \mathrm{C}$ de termorretificação.

Para a perda de massa, os resultados foram significativamente inferiores nos tratamentos $\mathrm{T}_{3}$ e $\mathrm{T}_{4}$. Dessa forma, o tratamento $\mathrm{T}_{2}\left(120^{\circ} \mathrm{C}-1 \mathrm{~h}\right)$ não se mostrou eficiente na inibição fúngica na madeira de E. tereticornis, considerando que a perda de massa foi significativamente igual à testemunha. 
Tabela 2 - Valores médios de massa residual (\%) e perda de massa (\%) da madeira de Eucalyptus tereticornis submetida a fungos causadores de podridão-branca.

Table 2 - Average values of residual mass (\%) and weight loss (\%) of Eucalyptus tereticornis wood subjected to white rot fungi.

\begin{tabular}{|c|c|c|c|c|}
\hline \multirow{3}{*}{ Fungos } & \multicolumn{4}{|c|}{ Variável: massa residual CV: $1,0 \%$} \\
\hline & \multicolumn{4}{|c|}{ Tratamentos } \\
\hline & $\mathrm{T} 1$ & $\mathrm{~T} 2$ & T 3 & $\mathrm{~T} 4$ \\
\hline T. versicolor & $97,94 \mathrm{Ab}$ & $99,16 \mathrm{Aa}$ & $99,82 \mathrm{Aa}$ & $99,94 \mathrm{Aa}$ \\
\hline G. applanatum & $96,52 \mathrm{Bb}$ & $98,24 \mathrm{Ab}$ & $99,48 \mathrm{Aa}$ & $100 \mathrm{Aa}$ \\
\hline \multirow{3}{*}{ Fungos } & \multicolumn{4}{|c|}{ Variável: perda de massa CV: $8,8 \%$} \\
\hline & \multicolumn{4}{|c|}{ Tratamentos } \\
\hline & T 1 & $\mathrm{~T} 2$ & T 3 & $\mathrm{~T} 4$ \\
\hline T. versicolor & $2,06 \mathrm{Ba}$ & $0,84 \mathrm{Ba}$ & $0,18 \mathrm{Ab}$ & $0,06 \mathrm{Ab}$ \\
\hline G. applanatum & $3,48 \mathrm{Aa}$ & $1,76 \mathrm{Aa}$ & $0,52 \mathrm{Ab}$ & $0,00 \mathrm{Ab}$ \\
\hline
\end{tabular}

* Médias seguidas por mesma letra maiúscula na coluna e minúscula na linha não diferem entre si, pelo teste de Tukey a 5\% de significância. $\mathrm{T}_{1}$ - durabilidade natural; $\mathrm{T}_{2}-120{ }^{\circ} \mathrm{C} 1 \mathrm{~h} ; \mathrm{T}_{3}-180{ }^{\circ} \mathrm{C} 4 \mathrm{~h} ; \mathrm{e} \mathrm{T}_{4}-120{ }^{\circ} \mathrm{C} 1 \mathrm{~h}+180{ }^{\circ} \mathrm{C} 4 \mathrm{~h}$.

Para a madeira de C. citriodora, todos os tratamentos tiveram a madeira classificada como altamente resistente a fungos xilófagos, com massa residual superior a $90 \%$ e perda de massa abaixo de $10 \%$, exceto no tratamento testemunha submetido ao apodrecimento pelo fungo $G$. applanatum, que foi classificado como resistente (Tabela 3 ). A interação entre espécies fúngicas e os tratamentos de termorretificação foi significativa $(p<0,05)$, e somente nos tratamentos $\mathrm{T}_{1}$ (testemunha) e $\mathrm{T}_{2}\left(120^{\circ} \mathrm{C}-1 \mathrm{~h}\right)$ houve diferença. No testemunha, a ação do fungo $G$. applanatum foi maior, levando a uma massa residual inferior. Entretanto, no $\mathrm{T}_{2}$ a menor massa residual foi encontrada sob a ação do fungo T. versicolor, indicando que a termorretificação em autoclave foi mais eficiente contra a outra espécie fúngica testada.
A perda de massa foi superior no fungo $G$. applanatum na madeira sem tratamento; no $\mathrm{T}_{3}(180$ $\left.{ }^{\circ} \mathrm{C}-4 \mathrm{~h}\right)$ e no $\mathrm{T}_{4}\left(120^{\circ} \mathrm{C}-1 \mathrm{~h}+180^{\circ} \mathrm{C} 4 \mathrm{~h}\right)$ não houve diferença entre os fungos, enquanto no $\mathrm{T}_{2}\left(120^{\circ} \mathrm{C}-\right.$ $1 \mathrm{~h}$ ) a perda de massa foi superior em $T$. versicolor. Os tratamentos mais eficientes na redução da perda de massa, quando a madeira foi exposta a ambos os fungos, foram $\mathrm{T}_{3}$ e $\mathrm{T}_{4}$.

Nos resultados colorimétricos, novamente a interação trifatorial não foi significativa, sendo os dados processados separadamente para cada espécie. Para E. tereticornis, a interação entre os fatores espécies de fungos e tratamentos térmicos também não foi significativa $(\mathrm{Fc}=1,88$ e $\mathrm{p}>0,005)$. Observando as diferenças colorimétricas na ação do ataque dos fungos

Tabela 3 - Valores médios de massa residual (\%) e perda de massa (\%) da madeira de Corymbia citriodora submetida a fungos causadores de podridão-branca.

Table 3 - Average values of residual mass (\%) and weight loss (\%) of Corymbia citriodora wood subjected to white rot fungi.

\begin{tabular}{lcccr}
\hline \multirow{2}{*}{ Fungos } & \multicolumn{4}{c}{ Variável: massa residual CV: 0,99\% } \\
\cline { 2 - 5 } & \multicolumn{4}{c}{ Tratamentos } \\
\cline { 2 - 5 } & $\mathrm{T} 1$ & $\mathrm{~T} 2$ & $\mathrm{~T} 3$ \\
\hline T. versicolor & $93,36 \mathrm{Ac}$ & $96,18 \mathrm{Bb}$ & $99,52 \mathrm{Aa}$ \\
G. applanatum & $88,63 \mathrm{Bb}$ & $98,15 \mathrm{Aa}$ & $99,24 \mathrm{Aa}$ & $99,35 \mathrm{Aa}$ \\
\hline \multirow{3}{*}{ Fungos } & \multicolumn{5}{c}{ Variável: perda de massa CV\%: 18,1\% } \\
\cline { 2 - 5 } & \multicolumn{4}{c}{ Tratamentos } \\
\cline { 2 - 5 } & $\mathrm{T} 1$ & $\mathrm{~T} 2$ & $\mathrm{~T} 3$ \\
\hline T. versicolor & $6,64 \mathrm{Ba}$ & $3,82 \mathrm{Ab}$ & $0,76 \mathrm{Ac}$ & $0,48 \mathrm{Ac}$ \\
G. applanatum & $11,38 \mathrm{Aa}$ & $1,85 \mathrm{Bb}$ & $1,63 \mathrm{Ab}$ & $0,65 \mathrm{Ab}$ \\
\hline
\end{tabular}

* Médias seguidas por mesma letra maiúscula na coluna e minúscula na linha não diferem entre si, pelo teste de Tukey a $5 \%$ de significância. $\mathrm{T}_{1}$ - durabilidade natural; $\mathrm{T}_{2}-120^{\circ} \mathrm{C} 1 \mathrm{~h} ; \mathrm{T}_{3}-180{ }^{\circ} \mathrm{C} 4 \mathrm{~h} ;$ e $\mathrm{T}_{4}-120{ }^{\circ} \mathrm{C} 1 \mathrm{~h}+180{ }^{\circ} \mathrm{C} 4 \mathrm{~h}$. 
em cada tratamento de E. tereticornis, verificou-se que, para a variável claridade $\left(\mathrm{L}^{*}\right)$, apenas no $\mathrm{T}_{2}(120$ ${ }^{\circ} \mathrm{C}-1 \mathrm{~h}$ ) ocorreu escurecimento da madeira após o ataque dos fungos. O menor valor $(27,8)$, mais escuro, foi observado no controle após a termorretificação no $\mathrm{T}_{4}$ $\left(180^{\circ} \mathrm{C}-4 \mathrm{~h}\right)$; e o maior valor, mais claro, no controle em $\mathrm{T}_{2}$ (Figura 1A).
Foi observada redução nos valores para a coordenada cromática $\mathrm{a}^{*}$ (Figura 1B) após o ataque de ambos os fungos causadores de podridão-branca em $\mathrm{T}_{2}\left(120^{\circ} \mathrm{C}\right.$ $-1 \mathrm{~h}$ ) em relação ao controle, indicando que o ataque dos fungos produziria efeito cromático mais próximo de verde. Para as variáveis $\mathrm{b} *$ e $\mathrm{C} *$ (Figura $1 \mathrm{CD}$, respectivamente), não houve diferenças significativas
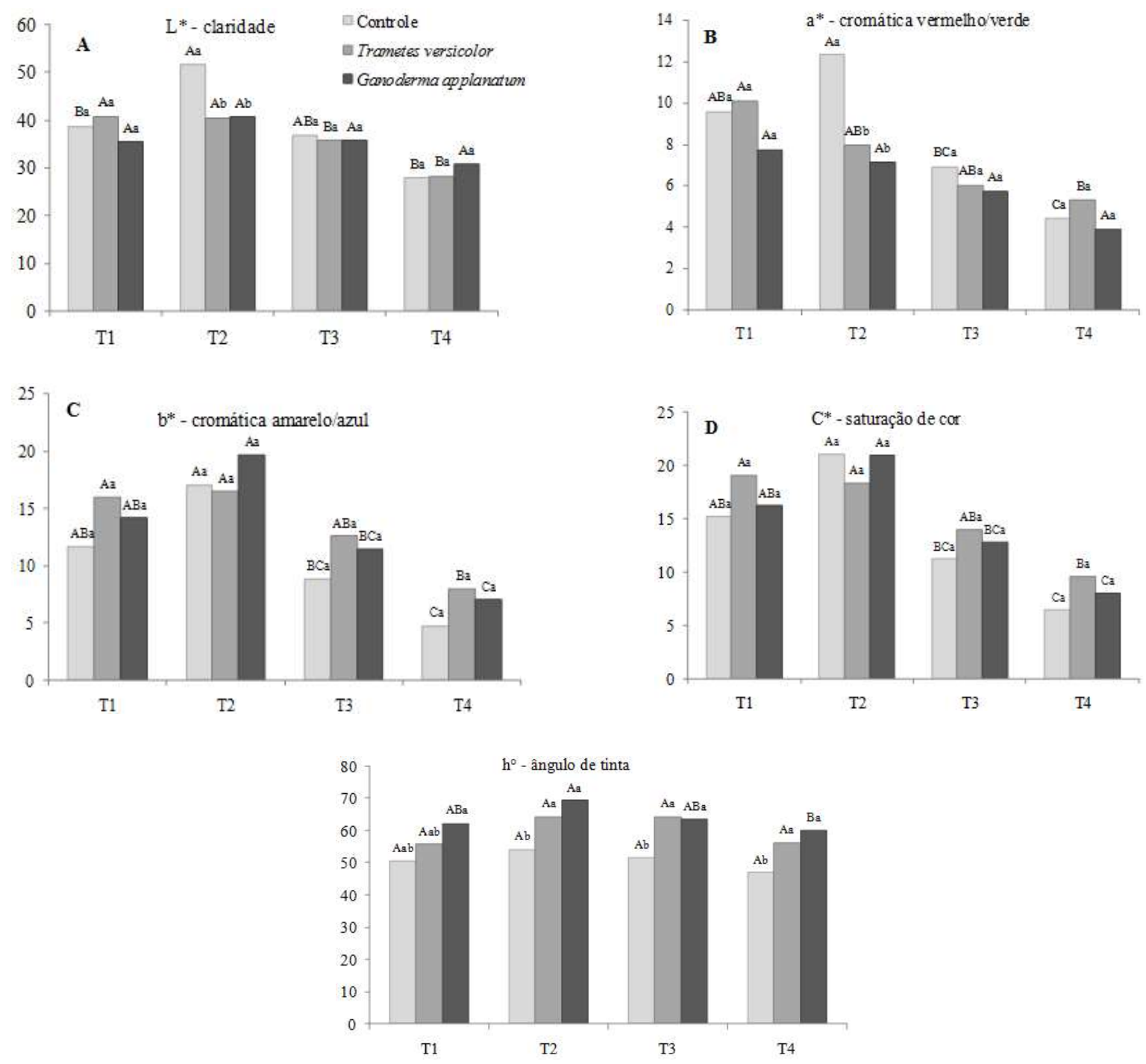

Figura 1 - Colorimetria de Eucalyptus tereticornis. Letras maiúsculas diferentes indicam diferenças entre os tratamentos térmicos e minúsculas, diferenças dentro de cada tratamento. $\mathrm{T}_{1}-$ controle; $\mathrm{T}_{2}-120^{\circ} \mathrm{C} 1 \mathrm{~h} ; \mathrm{T}_{3}-180^{\circ} \mathrm{C} 4$ $\mathrm{h} ; \mathrm{e} \mathrm{T}_{4}-120{ }^{\circ} \mathrm{C} 1 \mathrm{~h}+180{ }^{\circ} \mathrm{C} 4 \mathrm{~h}$.

Figure 1 - Colorimetry of Eucalyptus tereticornis. Different capital letters indicate differences between the heat treatments and lowercase indicate differences within each treatment. $T_{1}-$ control, $T_{2}-120^{\circ} \mathrm{C} 1 \mathrm{~h} ; \mathrm{T}_{3}-180^{\circ} \mathrm{C} 4 \mathrm{~h}$; and $\mathrm{T}_{4}-120^{\circ} \mathrm{C} 1 \mathrm{~h}+180^{\circ} \mathrm{C} 4 \mathrm{~h}$.

Revista Árvore, Viçosa-MG, v.40, n.1, p.135-145, 2016 
quando comparadas com o ataque dos fungos e com a madeira sem exposição ao agente xilófago.

Comparando apenas os tratamentos térmicos, houve tendência à diminuição da variável $b^{*}$ após a termorretificação, tanto para madeira exposta quanto para madeira não exposta aos fungos apodrecedores. O gráfico para $C^{*}$ (Figura 1D) foi semelhante ao de $b^{*}$ (Figura 1C) e, como a primeira variável depende dos valores de $\mathrm{a}^{*} \mathrm{e} \mathrm{b}^{*}$, verificou-se que a matriz cromática amarelo/azul foi a que teve maior influência sobre a saturação. Para $\mathrm{h}^{\circ}$, a madeira não exposta aos fungos (controle) apresentou valores significativamente inferiores dessa variável em $\mathrm{T}_{2}, \mathrm{~T}_{3}$ e $\mathrm{T}_{4}$

Na madeira de C. citriodora, a interação entre os fatores espécies de fungos e tratamentos térmicos foi significativa $(\mathrm{Fc}=18,06 \mathrm{e} \mathrm{p}<0,005)$. A variável claridade $\mathrm{L}^{*}$ foi diferente significativamente entre as espécies de fungos inoculados em $\mathrm{T}_{1}$ (testemunha), $\mathrm{T}_{2}\left(120^{\circ} \mathrm{C}-1 \mathrm{~h}\right)$ e $\mathrm{T}_{4}\left(180^{\circ} \mathrm{C}-4 \mathrm{~h}\right)$, em que em $\mathrm{T}_{1}$ a madeira inoculada com $G$. applanatum foi a mais clara $\mathrm{e}, \mathrm{em} \mathrm{T}_{2}$, a que apresentou menor valor, ou seja, a mais escurecida; $\mathrm{em}_{4}$, a madeira sem inoculação de fungos foi a mais clara (Figura 2A). Ainda com relação a essa mesma variável, os tratamentos $\mathrm{T}_{3} \mathrm{e}_{4}$ tornaram a madeira mais escura, já que esses tratamentos promoveram o escurecimento tanto da madeira não submetida à ação dos fungos apodrecedores quanto daquela que foi submetida à inoculação de ambos.

A mesma tendência de redução foi observada na variável $\mathrm{a}^{*}$, indicando uma aproximação da cromática verde tanto para controle quanto para a madeira exposta à termorretificação, especialmente nos tratamentos $\mathrm{T}_{3}$ e $\mathrm{T}_{4}$ (Figura 2B). Redução significativa dos valores também foi observada na variável $b^{*}$, indicando aproximação da cromática azul, com menores valores registrados em $\mathrm{T}_{3}$ e $\mathrm{T}_{4}$, embora não tenham sido verificadas diferenças estatísticas entre os corpos de prova utilizados como controle e aqueles submetidos ao apodrecimento acelerado com os dois fungos causadores de podridão-branca (Figura 2C). O gráfico de saturação de cor $C^{*}$ (Figura 2D) assemelha-se ao da cromática amarelo/azul b*, indicando maior influência da cromática azul na colorimetria da espécie $C$. citriodora. Os menores valores de ângulo de tinta $\mathrm{h}^{\mathrm{o}}$ foram observados em $\mathrm{T}_{3}$ e $\mathrm{T}_{4}$ na madeira inoculada e não inoculada com fungos apodrecedores, e também houve diferenças significativas nessa variável entre os tratamentos de inoculação, em que a madeira que sofreu ataque de organismo xilófago foi estatisticamente superior em todos os tratamentos térmicos (Figura 2E).

\section{DISCUSSÃO}

Em relação à massa específica, Tomazello Filho et al. (2005) encontraram os valores de $0,73 \mathrm{~g} / \mathrm{cm}^{3} \mathrm{e}$ $0,66 \mathrm{~g} / \mathrm{cm}^{3}$ para C. citriodora e E. tereticornis, respectivamente, em árvores com idade aproximada de 16 anos. Os valores encontrados por esses autores foram diferentes dos obtidos neste estudo, entretanto é importante ressaltar que os corpos de prova deste trabalho foram oriundos de árvores de idade aproximada de 60 anos.

De modo geral, em ambas as espécies os tratamentos térmicos, realizados em condição de seca e de temperaturas superiores, foram mais eficazes na redução da perda de massa da madeira, quando expostas a fungos causadores da podridão-branca. Esses resultados vão de encontro às afirmações de Weiland e Guyonnet (2003) de que, quanto maior o tempo de exposição e a temperatura do tratamento, melhor a resistência do produto final ao fungo deteriorador. Isso porque a termorretificação sob altas temperaturas, conforme já mencionado por Silva et al. (2008), podem gerar novos extrativos que podem atuar como fungicidas, modificam quimicamente a madeira e, ainda, degradam as hemiceluloses que são fontes de alimento para os fungos.

Com relação à durabilidade natural de madeira de espécies de eucalipto, Oliveira et al. (2005) observaram que a madeira de C. citriodora, E. tereticornis, $E$. paniculata, E. pilularis, E. cloeziana, E. urophylla e $E$. grandis teve perdas de massa inferiores a $10 \%$ quando submetidas ao apodrecimento acelerado pelo fungo causador da podridão-parda Gloephyllum trabeum e E. tereticornis foi mais resistente que $C$. citriodora. Esse último resultado foi confirmado neste estudo para o fungo apodrecedor Ganoderma applanatum, que provocou maior perda de massa em C. citriodora $(11,375 \%)$ em relação a E. tereticornis $(3,482 \%)$ na testemunha, embora a interação do fator espécies de madeira não tenha sido significativa neste estudo.

Comparando os resultados de parâmetros colorimétricos para as espécies estudadas com outras já pesquisadas, Zanuncio et al. (2014) observaram valores entre 65,3 e 32,3 para claridade e constataram que, à medida que os tratamentos de termorretificação para 

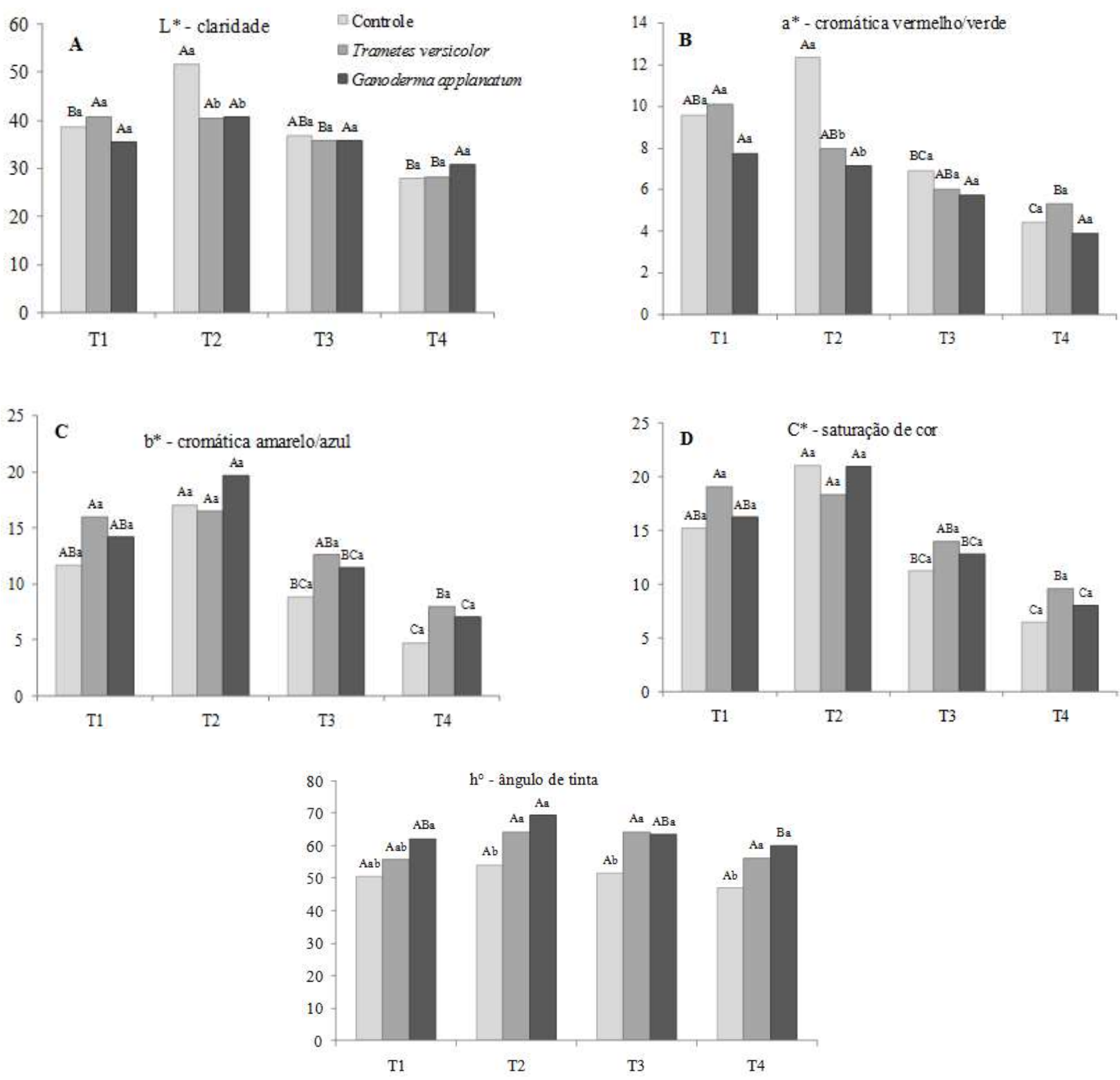

Figura 2 - Colorimetria de Corymbia citriodora. Letras maiúsculas diferentes indicam diferenças entre os tratamentos térmicos e minúsculas, diferenças dentro de cada tratamento. $\mathrm{T}_{1}$ - controle; $\mathrm{T}_{2}-120^{\circ} \mathrm{C} 1 \mathrm{~h} ; \mathrm{T}_{3}-180{ }^{\circ} \mathrm{C} 4 \mathrm{~h}$; e $\mathrm{T}_{4}-$ $120{ }^{\circ} \mathrm{C} 1 \mathrm{~h}+180{ }^{\circ} \mathrm{C} 4 \mathrm{~h}$.

Figure 2 - Colorimetry of Corymbia citriodora. Different capital letters indicate differences between the heat treatments and lowercase indicate differences within each treatment. $T_{1}-$ control, $T_{2}-120^{\circ} \mathrm{C} 1 \mathrm{~h} ; \mathrm{T}_{3}-180^{\circ} \mathrm{C} 4 \mathrm{~h}$; and $\mathrm{T}_{4}-120^{\circ} \mathrm{Cl} \mathrm{h}+180^{\circ} \mathrm{C} 4 \mathrm{~h}$.

Eucalyptus grandis se intensificavam, ocorria escurecimento da madeira, tal qual observado neste estudo. Cademartori et al. (2014a) também observaram o escurecimento da madeira de E. grandis e E. saligna progressivo em função do aumento da temperatura de tratamento de termorretificação. Segundo esses autores, a formação de aldeídos e fenóis é observada quando a madeira é exposta a altas temperaturas e, consequentemente, os compostos de coloração são formados após as reações químicas. Mitsui et al. (2004) 
afirmaram que a redução da variável claridade em madeira de Criptomeria japonica é atribuída aos extrativos e componentes de baixo peso molecular produzidos pela lignina e hemicelulose.

Essa mudança nos parâmetros colorimétricos após o ataque de fungos apodrecedores da madeira já foi verificada por Costa et al. (2011), submetendo as madeiras de Simarouba amara (marupá) e Carapa guianensis (andiroba) ao ataque de Trametes versicolor e Gloephyllum trabeum. Na madeira de marupá houve escurecimento após o ataque de ambos os fungos, enquanto na de andiroba ocorreu escurecimento apenas após o ataque de G. trabeum.

Algumas alterações colorimétricas podem não ser desejáveis, como tornar a madeira mais esverdeada ou azulada (representadas pelos parâmetros cromáticos $\mathrm{a}^{*}$ e $\mathrm{b}^{*}$ ). Já o escurecimento pelos tratamentos de termorretificação pode ser benéfico, considerando que seria possível evitar a utilização de tintas e aplicações mais tóxicas para escurecimento de madeiras, a fim de torná-las com aspecto de madeiras nobres, considerando que muitas madeiras de espécies nativas consideradas nobres possuem tons mais escurecidos em relação às espécies de eucalipto.

Os resultados dos parâmetros colorimétricos observados em E. tereticornis repetiram-se em $C$. citriodora neste estudo. Cademartori et al. (2012) também observaram queda na matriz amarelo (b*) de 20,57 para 17,69 e 10,93 na madeira de E. grandis a $180^{\circ} \mathrm{C}$ e 240 ${ }^{\circ} \mathrm{C}$ por $4 \mathrm{~h}$, respectivamente. Compostos cromóforos associados às ligninas e aos extrativos conferem cor amarelada à madeira, entretanto o tratamento térmico pode degradar esses compostos, reduzindo, assim, essa matriz (PINCELLI et al., 2012).

Considerando o parâmetro ângulo de tinta $\left(\mathrm{h}^{\circ}\right)$, Pincelli et al. (2012) relataram tendência inversa ao deste trabalho para E. saligna, com aumento do ângulo de tinta após o tratamento térmico; já Cademartori et al. (2012) relataram redução dessa variável em E. grandis.

O tratamento térmico da madeira tem ganhado destaque como método eficaz para melhorar sua estabilidade dimensional e resistência biológica a organismos. Fazendo uma análise geral do comportamento da madeira de E. tereticornis e C. citriodora em relação às variáveis colorimétricas, observaram-se mudanças significativas nos parâmetros, especialmente em função dos tratamentos térmicos, e poucas mudanças foram verificadas nesses mesmos parâmetros, em comparação com a madeira com ou sem submissão ao apodrecimento acelerado, em ambas as espécies dos fungos da podridãobranca testados.

\section{CONCLUSÕES}

O Eucalyptus tereticornis é considerado altamente resistente aos fungos causadores da podridão-branca (Trametes versicolor e Ganoderma applanatu).

C. citriodora é considerado resistente apenas contra Ganoderma applantum.

Os tratamentos térmicos realizados a $180^{\circ} \mathrm{C}$, condição seca, são eficientes na redução da perda de massa de madeira atacada pelos fungos $T$. versicolor e G. applanatum

O tratamento térmico realizado a $120^{\circ} \mathrm{C}$, condição úmida, é ineficiente para aumento da durabilidade natural da madeira de E. tereticornis e C. citriodora.

A termorretificação realizada a $180^{\circ} \mathrm{C}$, na condição seca, apresenta-se como alternativa para a alteração das cores da madeira de E. tereticornis e C. citriodora, visando ao seu escurecimento, ou seja, redução da variável claridade $\left(\mathrm{L}^{*}\right)$, e à queda das matrizes vermelho (a*) e amarelo (b*).

\section{AGRADECIMENTOS}

À Coordenação de Aperfeiçoamento de Pessoal de Nível Superior (CAPES), pela concessão da Bolsa PNPD - Institucional (Programa Nacional de PósDoutorado) ao primeiro autor por ocasião da realização deste trabalho.

\section{REFERÊNCIAS}

ARAÚJO, H.J.B.; MAGALHÃES, W.L.E.; OLIVEIRA, L.C. Durabilidade de madeira de eucalipto citriodora (Corymbia citriodora (Hook.) K.D. Hill \& L.A.S. Johnson) tratada com CCA em ambiente amazônico. Acta Amazônica, v.42, n.1., p.49-58, 2012.

AMERICAN SOCIETY FOR TESTING AND MATERIALS - ASTM. Standard test method of accelerated laboratory test of natural decay resistance of woods. ASTM D 2017. Annual book of ASTM standards. West Conshohocken: 2005.

Revista Árvore, Viçosa-MG, v.40, n.1, p.135-145, 2016 
VILLAS BÔAS, O.; MAX, J.C.M.; MELO, A.C.G. Crescimento comparativo de espécies de Eucalyptus e Corymbia no município de Marília, SP. Revista do Instituto Florestal, v.21, n.1, p.63-72, 2009.

BRAND, M.A.; ANZALDO, J.; MORESCHI, J.C. Novos produtos para o tratamento preservante da madeira. "Perspectivas da pesquisa e utilização". Floresta, v.36, n.1, p.129-137, 2006.

CADEMARTORI, P.H.G.; SCHNEID, E.; GATTO, D.A.; BELTRAME, R.; STANGERLIN, D.M. Modification of static bending strength properties of Eucalyptus grandis heat-treated wood.

Materials Research, v.15, n.6, p.922-927, 2012.

CADEMARTORI, P.H.G.; MATTOS, B.D.; MISSIO, A.L; GATTO, D.A. Colour responses of two fastgrowing hardwoods to two-step steamheat treatments. Materials Research, v.17, n.2, p.487-493, 2014a.

CADEMARTORI, P.H.G.; MISSIO, A.L; MATTOS, B.D.; SCHNEID, E.; GATTO, D.A. Physical and mechanical properties and colour changes of fastgrowing Gympie messmate wood subjected to two-step steam-heat treatments. Wood

Material Science \& Engineering, v.9, n.1, p.1-9, 2014b.

COSTA, M.A.; COSTA, A.F.; PASTORE, T.C.M.; BRAGA, J.W.B.; GONÇALEZ, J.C. Caracterização do ataque de fungos apodrecedores de madeiras através da colorimetria e da espectroscopia de infravermelho. Ciência Florestal, v.21, n.3, p.567-577, 2011.

DELUCIS, R.A.; GATTO, D.A.; CADEMARTORI, P.H.G.; MISSIO, A.L; SCHNEID, E. Propriedades físicas da madeira termorretificada de quatro folhosas. Floresta e Ambiente, v.21, n.1, p.99-107, 2014.

ESTEVES, B.M.; PEREIRA, H.M. Wood modification by heat treatment: A Review. Bioresources, v.4, n.1, p.370-404, 2009.

ESTEVES, B.; GRAÇA, J.; PEREIRA, H. Extractive composition and summative chemical analysis of thermally treated eucalypt wood.

Holzforschung, v.62, n.3, p.344-351, 2008.
FERREIRA, D.F. Sisvar: A computer statistical analysis system. Ciência e Agrotecnologia, v.35, n.6, p.1039-1042,2011.

KAMDEM, D.P.; PIZZI, A.; JERMANNAUD, A. Durability of heat-treated wood. Holz als Roh-und Werkstoff, v.60, p.1-6, 2002.

MILITZ, R. Heat treatment technologies in Europe: scientific background and technological state-of-art. In: CONFERENCE ON ENHANCING THE DURABILITY OF LUMBER AND ENGINEERED WOOD PRODUCTS, 1., 2002, Orlando. Proceedings... Madison: Forest Products Society, 2002. p.1-19.

MITSUI, K. hanges in the properties of lightirradiated wood with heat treatment. Part 2. Effect of lightirradiation time and wavelength. Holz als Roh- und Werkstoff, v.62, n.1, p.23-30, 2004.

NUOPPONEN, M.; VUORINENA, T.; JÄMSÄB, S.; VIITANIEMIB, P. Thermal Modifications in Softwood Studied by FT IR and UV Resonance Raman Spectroscopies. Journal of Wood Chemistry and Technology, v.24, n.1, p.1326, 2004.

OLIVEIRA, J.T.; TOMASELLO, M.; SILVA, J.C. Resistência natural da madeira de sete espécies de eucalipto ao apodrecimento. Revista Árvore, v.29, n.6, p.993-998, 2005.

PINCELLI, A.L.P.S.M.; DE MOURA, L.M., BRITO, J.O. Effect of thermal rectification on colors of Eucalyptus saligna and Pinus caribaea woods. Maderas. Ciencia y Tecnologia, v.14, n.2, p.239-248, 2012.

POUBEL, D.S.; GARCIA, R.A.; SANTOS, W.A.; OLIVEIRA, G.L.; ABREU, H.S. Efeito da termorretificação nas propriedades físicas e químicas da madeira de Pinus caribaea. Cerne, v.19, n.3, p.391-398, 2013.

SILVA, M.R.; MACHADO, G.O.; CALIL JUNIOR, C. Efeito do tratamento térmico nas propriedades mecânicas de Pinus elliotti. In: ENCONTRO BRASILEIRO EM MADEIRA E ESTRUTURAS DE MADEIRA, 11., 2008, Londrina. Anais... Londrina: UEL - EBRAMEM, 2008. 
TJEERDSMA, B.F.; MILITZ, H. Chemical changes in hydrothermal treated wood: FTIR analysis of combined hydrothermal and dry heat-treated wood. European Journal of Wood and Wood Products, v.63, n.2, p.102-111, 2005.

TOMAZELLO FILHO, M.; HELLMEISTER, J.C.; OLIVEIRA, J.T.S. Variação do teor de umidade e da densidade básica na madeira de sete espécies de eucalipto. Revista Árvore, v.29, n.1, p.115-127, 2005.
WEILAND, J.; GUYONNET, R. Study of chemical modifications and fungi degradation of thermally modified wood using DRIFT spectroscopy. Holz als Roh- und Werkstoff, v.61, p.216-220, 2003.

ZANUNCIO, J.A.V.; FARIAS, E.S.; SILVEIRA, T.A. Termorretificação e colorimetria da madeira de Eucalyptus grandis. Floresta e Ambiente, v.21, n.1, p.85-90, 2014. 